\title{
Study on Artistic Characteristics of Erhu Concertos before Reform and Opening-up
}

\author{
Jindi Zhang \\ Art College, Shandong University, Weihai, 264209, China
}

Keywords: Erhu; Art

\begin{abstract}
The Erhu music development history in the second half of the $20^{\text {th }}$ century is also a history reflecting the reforms and developments of new China since its establishment. After reform and development in the first half century, Erhu music entered the period of great prosperity and development in the second half century. The names of Erhu music tracks could reflect and allude to the social and political situations at that time. The development of Chinese music in this period was restricted and influenced by the politics. The Erhu concertos which appeared in the 30s almost disappeared in the later dozens of years and were replaced by revolution tracks, movement tracks and fighting tracks in praise of the era.
\end{abstract}

\section{Artistic characteristics of Erhu concertos}

After establishment of the new China, the emancipated people, which became the owners of the country, were highly enthusiastically building the socialism. The music industry also answered the call, developing the creation of Erhu tracks in the premise of excavating, systemizing and studying folk music. The folk music's influence on original works at that time was far-reaching and the creation of Erhu concertos was still in the emerging and exploration phase.

The lengths of Erhu music works in this period were generally short and crack, with a playing time of 5 or 6 minutes mostly. The playing time of concerto works was often about 10 minutes. There was no large-scale works in this period.

At the end of 50s and at the beginning of 60s, the nationwide activities for investigation and research of literature and art largely boosted the creation and there was once a small vigorous and prosperous climax in the creation with Chinese traditional music instruments; many works with Chinese traditional music instruments with strong response and wining universal praises were created.

\section{Expansion of Erhu performance skills and acoustic effect}

In 1944, Zhang Zirui, a student of Nanjing State-run Music Technical College, imitated the violin's application of metallic strings to change the Erhu's strings into metallic strings, which not only changed the tone brightness, volume size and other acoustic effects of Erhu but also expanded Erhu's performance techniques and artistic expressive force, largely boosting the later creation of Erhu music.

In the 60s, Central Conservatory of Music and other academies, literature and art groups, research institutions and musical instrument factories cooperated to formulate the unified standards for the fabrication specifications and quality of Erhu, reformed and reorganized the high-pitch, alto, bass and double bass instruments and determined the structural system pattern of orchestral band with the combination of flute blowing, Erhu and violin playing and drum beating. The string instrument Erhu in the alto voice acted as the principal music instrument in the string team, which is different from the violin acting as the principal music instrument in the high-pitched voice in the Western band. The band's organization system and acoustic effect were strengthened, further propelling the concerto between Erhu and band as well as the acoustic effect.

The utilization of metallic strings expanded the valid range of Erhu performance, and the frequent use of high-range timbre increased the application of large-amplitude holding position variation skill and the difficulty in mastering the high-range timbre skill. Performance skills were richer with the 
enrichment of Erhu music works, and music styles were diversified; the emotional expression were not limited by euphemistic and plaintive silk string performance skills any more; metallic string performance art expressed the optimistic and lively spirits of the new age, and new performance skills emerged at the right moment. The use of fast bow became a highlight of skills; compared with those works before the foundation of PRC, the frequent use of fast bow significantly speeded up the music, and the performance skills of fast bow also gradually increased the requirements on definition and graininess as well as the difficulty in string shifting and holding position variation. For example: in the Sanmen Gorge Rhapsody, the fast bow virtuosity with semiquaver was a new challenge for Erhu performance skills, which surpassed the Erhu performance skills of Liu Tianhua period.

For traditional programs, the Erhu timbres were required to be much brighter, more relaxed, more splendid and more flowable, and the techniques were closer to bowing characteristics of violin; the sense of bowing speed and variation presentation were added.

The large arpeggio, septuplet, dodecuplet, and other skills got increasingly complicated, and the musical composition Sanmen Gorge Rhapsody represented the technique height at that time.

The variation of pentatonic scale, equally tempered diationic scale, and interval relation became more difficult, and the application of modulation, meter and rhythmic variation was more frequent.

The left-hand performance techniques are rich and colorful; in the performance, the performance techniques of rolling rubbing, pressing rubbing, sliding rubbing, embracing rubbing, vibrato, portamento, finger padding, erasure, etc., and the performance techniques of old lute, Chinese lute and violin were combined and utilized in Erhu performance art.

The right-hand bowing techniques were diversified, including martele, piccato, ricochet, bow drawing, pizzicato, striking, etc.; simulated audio technology of bowing tension referenced other sister arts, and even surpassed their development.

\section{Integration of western composition skills and Chinese aesthetics}

In the $20^{\text {th }}$ century, Erhu music composition was an emerging specialty and developed very fast, and the creation of Erhu concertos pushed the Erhu to the development peak. In this stage, Erhu developed from accompaniment to solo, and took the lead in the concerto. The development of Erhu desired for further requirements on works.

The most important characteristics of Erhu concertos that were different from solo composition was that it would consider not only the development of Erhu melody theme, but also the harmonious development between accompanied instruments and presided instruments as well as the dialogue concertato relationship. Thus, the writer needs to understand the performance and skills of western and Chinese instruments, the western and Chinese composition performance and skills, the aesthetic appreciation and expression habits of western and Chinese cultures, which is the key point to check whether the writer can rein and integrate western and Chinese cultures for creation.

Chinese aesthetic appreciation is the extension of horizontal linear thinking, while western composition techniques are the superposition of longitudinal harmony and polyphony. How to integrate them is a technique and even a kind of culture. In this aspect, Mr. Liu Wenjin is a master. For example, in the North Henan Ballad:

The first paragraph "descriptively": The first sentence is an eight-section square changing and concluding phrase, starting with gentle note 3 and ending with penetrating note 6; the gentle note 3 is the third note of primary chord of major and the fifth note of primary chord of minor as well as the radical of dominant chord, and the penetrating note 6 is the key note of minor. There are major and minor colors and the national mode color; the mode and tonality are integrated.

The six-note pattern 123567 was used as the melody, and the leading note 7 was used on the "changing" of the fifth section; thus, the first inversion dominant seventh chord was used. The scales of melody excluded the scale 4, and only the dual dominant seventh chords of falling tone 3(diatonic tone) were used in the eighth section for the harmonic progression; the chord and melody were integrated. 
The harmonic progression used the broken chord, including the linear summation tone echo and the harmonic sound support. With the singing melody, full of stereo feeling was reflected in the gentleness, and the horizontal and longitudinal integration was seamless.

The second paragraph "cheerfully": It starts with the two four-bar repeated phrases; the countermelody of left-hand low vocal part was formed by vocal reduction of main melody; the high vocal melody of Erhu and the left-hand low vocal part of piano echo each other, and the right-hand harmony was fully filled between the two parts. The active chord accompaniment starting from the right-hand latter-half beat and the left-hand single tone emerged alternatively, generating light and clear emotional feelings. The two phrases from the $9^{\text {th }}$ bar to the $18^{\text {th }}$ bar adopted the simulated question-answering and dialogic techniques of piano to reproduce the melody variation at the piano voice part, generating the sound effect of dialogue between Erhu and piano.

\section{Artistic and literal characteristics of forms and contents of works}

(1) Works have characteristics of being programmatic, concrete, spatial and political

(2) Western-Chinese mixing, form-meaning integration

\section{Development of Erhu concerto creation and performance art}

Such expression form of Erhu and band reappeared after 1944, showing the development potential of Erhu; the Erhu performance art would have a broader development prospect.

The "Shanghai Spring" Erhu competition held in 1963 promoted the creation of Erhu concertos, and new works were unprecedentedly prosperous, which became the first leap in the Erhu art development history after the establishment of the new China. The North Henan Ballad and the Sanmen Gorge Rhapsody created by Mr. Liu Wenjin generated a huge response, which pushed the Erhu music creation to the height of professionalization.

The lyricism of the North Henan Ballad was strengthened, and the writer adopted the variation with decoration to change and develop the theme language and put the delicate emotional variation and the mood bedding and expansion to good use in the phrase language, so as to slowly expand and push the emotion to a high level with the plot. Such exquisite and relaxed emotion control significantly promoted the Erhu performance techniques and enhanced the artistic processing of Erhu works.

The Sanmen Gorge Rhapsody presented the majestic momentum, and broke through the weak and mellow sound production characteristics of Erhu as well as the limitation to small subjects; the works expanded the momentum and powerful performance skills of Erhu, and the performer's control ability was also improved. From this works, people had a brand new understanding of the performance potential of Erhu, and confirmed the capacity of Erhu to control large works.

The performer Wang Guotong was the first person performing the two epoch-making works and became the symbol of height for fast Erhu bowing skills. Good works can be shown to audiences only with good interpretation by the performer, and this is the significance of recreation. Wang Guotong could easily control the fast bowing skills and had obtained the epochal breakthrough, which was certainly related to his practice in fast bowing skills of Hora Staccato, Flight of the Bumble Bee, etc. during his school days.

We could say that it was Wang Guotong who broke the path of Erhu fast-bowing art and made unremitting efforts for spreading and promoting the fast bowing art.

\section{Representative works and figures}

On the World Bowstring Music Conference held in America on July 4, 2008, American members submitted the memorandum of the conference and thought that: "The birth of Mr. Liu Wenjin was the turning point of Erhu creation; similarly, the birth of Mendelssohn was the turning point of violin creation; they referred to the Sanmen Gorge Rhapsody and the Beethoven \& Mendelssohn Violin Concertos." 
Li Wenjin often listened to the folk music in his hometown Anyue City in Henan province from his childhood to the middle school period: Pangtzu of Honan, musical tune, blowing song, etc., and the original folk art was his "abecedarian". During the middle school period, he was the leader and conductor of the after-class folk band, and learned the performance of Erhu, Gaohu, flute, Qinqin, accordion and many other instruments. He studied the folk music composition in Central Conservatory of Music in 1956. The folk music had a far-reaching influence on Liu Wenjin's music creation.

The Sanmen Gorge Rhapsody made many breakthroughs in Erhu creation, and these breakthroughs were the results of the results of mutual fusion, penetration and organic combination between western and Chinese cultures. Breakthroughs in composition theme and form application, Erhu performance skills, Erhu artistic tension presentation, fusion between Erhu and piano, and especially the fast bowing techniques, become a cross in the Erhu art development history; it became an epoch-making works. These breakthrough innovations surpassed the technical height of Erhu music that was created by Mr. Liu Tianhua and was maintained for thirty years.

The two works North Henan Ballad and the Sanmen Gorge Rhapsody, one of which was gentle and the other was rigid, were praised as twin sisters in Erhu works, are still the commonly used works by Erhu learners and in concerts, and are universally acknowledged in the field as another milestone of Erhu works after Liu Tianhua period.

\section{Teaching and theoretical research for Erhu concertos}

After establishment of new China, all conservatories were founded and the music education and theoretical research for Erhu Major were developed unprecedentedly. The young concert performers showing their talents took the heavy responsibility of education and, meanwhile, played the roles of creators and performers. Therefore, they showed their own characteristic styles and features in concerts and teaching.

After the establishment of new China, the creation and arrangement of Erhu music had the diversified development tendency. The musicians of old generation mainly excavated traditional music and the musicians of new generation created and arranged Erhu music with the emerging elements to express their praises to the new era and new life. Pull the Camel, arranged by Zeng Xun in 1954, is the first piece of Erhu music by using the fast bow technique and was be all fashion in the 1960s. later, the use of period of fast bow in Erhu music was very popularized. The period of fast bow in Sanmen Gorge Rhapsody pushed the fast bow to a technical height.

Continuously innovative techniques of expression of music pieces stimulated the rapid development of Erhu performance techniques and motivated the professional, scientific and systematic development of Erhu technical training and Erhu teaching and teaching methods. In 1960, the first meeting for Erhu teaching in conservatories was convened nationwide. In 1961, the first meeting for Erhu teaching materials in institutions of higher learning was convened nationwide. The Erhu etudes and Erhu teaching materials were developed fast in this period. Selection of Erhu Music, compiled with the assistance of Lin Xinming of Shanghai Conservatory of Music, was published by Shanghai Literature and Art Publishing House in 1963, publishing Sun Wenming's music piece The Pathetic Melody etc., systemized and notated by Lin Xinming. Zhang Rui of Nanjing University of the Arts have the music collections, Zhang Rui Erhu Etudes and Yuhua Music Collection etc.; Selections of Erhu Etudes and Erhu Teaching Materials created by Wang Guotong and Zhang Shao of Central Conservatory of Music; Erhu Broadcasting Lecture (Shanghai Literature and Art Publishing House in 1959), Selections of Erhu Solos (Shanghai Literature and Art Publishing House)of Zhang Shao.

In the period with rapid development of fast bow technique, we need to particularly mention Wang Guotong, a Erhu concert performer and educator, and his huge contributions to this field. In 1961, he arranged Erhu Fast Bow Practice, Erhu Scale Practice and Erhu Fingering Technique Practice, which were the earliest teaching materials of new China; in the 1970s, he and Zhang Shao jointly arranged and published the Selections of Erhu Etudes which influenced many generations and became a 
invisible power to popularize and improve Erhu. He made huge contributions to the systematization and standardization construction of Erhu teaching. The "Erquan String”, "Fangyuan Erhu" and "Erhu with eight flat angles" developed and made by him became the regular Erhus, with the "metallic pull rod shaft" and improved Erhu bow etc. embodying the best tone of Erhu.

Wang Guotong made important contributions to the Erhu performance, compilation of teaching materials, works production, talent training, Erhu reform and other fields. The fast bow technique is the biggest motivation for follow-up Erhu technical development. 28 pieces among the 32 pieces of works in Selections of Erhu Music from 1949 to 1979 were played with fast bow, which became the Erhu technique popularly used in that period.

\section{Conclusion}

Generally speaking, the development features before the reform and opening up altered the creation with the traditional music structure of changing and conclusion of Liu Tianhua's period and most pieces of music applied the structural form of European ternary form. The length of music belonged to small-scale muse. The application of fast bow is the expressive way of new language. Compared with the last period, the music color looked more bright, brisk and vigorous. There were new standard requirements for professional education for the etudes of fast bow technique training.

\section{References}

[1] Liu Zaisheng, Opening, Surpassing and Exploration - "Trilogy" of Wang Guotong's Erhu Art Career, Journal of Central Conservatory of Music, 2011(1): 85 